\title{
Identification of the novel localization of tenascinX in the monkey choroid plexus and comparison with the mouse
}

\author{
K. Imura and I. Sato
}

Department of Anatomy, School of Life Dentistry at Tokyo, The Nippon Dental University, Japan

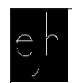

(C)2009 European Journal of Histochemistry

Tenascin- $X(T n-X)$ belongs to the tenascin family of glycoproteins and has been reported to be significantly associated with schizophrenia in a single nucleotide polymorphism analysis in humans. This finding indicates an important role of $\mathrm{Tn}-\mathrm{X}$ in the central nervous system (CNS). However, details of Tn-X localization are not clear in the primate CNS. Using immunohistochemical techniques, we found novel localizations of Tn-X in the interstitial connective tissue and around blood vessels in the choroid plexus (CP) in macaque monkeys. To verify the reliability of $\mathrm{Tn}-\mathrm{X}$ localization, we compared the Tn-X localization with the tenascin-C (Tn-C) localization in corresponding regions using neighbouring sections. Localization of $\mathrm{Tn}-\mathrm{C}$ was not observed in CP. This result indicated consistently restricted localization of $\mathrm{Tn}-\mathrm{X}$ in $\mathrm{CP}$. Comparative investigations using mouse tissues showed equivalent results. Our observations provide possible insight into specific roles of $\mathrm{Tn}-\mathrm{X}$ in $\mathrm{CP}$ for mammalian CNS function.

Key words: tenascin- $X$, choroid plexus, monkey, mouse, Ehlers-Danlos syndrome, schizophrenia.

Correspondence: Kosuke Imura,

Department of Anatomy, School of Life Dentistry at Tokyo, The Nippon Dental University, 1-9-20 Fujimi, Chiyoda-ku, Tokyo 102-8159, Japan.

Tel. +81.3.3261.8311.

Fax +81.3.3264.

E-mail: imurako@tky.ndu.ac.jp

Paper accepted on September 30, 2009

European Journal of Histochemistry 2009; vol. 53 issue 4 (October-December): 225-232
T he tenascins ( $\mathrm{Tn})$ are a family of four glycoprotein members - tenascin- $C(\mathrm{Tn}-\mathrm{C})$, tenascin-R ( Tn-R), tenascin-W $(T n-W)$ and tenascin-X $(T n-X)$ - found diversely in the extracellular matrix of vertebrate organs (Hsia and Schwarzbauer, 2005; Tucker and ChiquetEhrismann, 2009). Important functions of Tn have been investigated in developmental cell adhesion modulation and pathological conditions such as wound healing and tumourigenesis (Adams and Watt, 1993; Hsia and Schwarzbauer, 2005; Tucker and Chiquet-Ehrismann, 2009). Tn-C and Tn-R are prominent in the nervous system and play a role in the development of neurite outgrowth and postnatal synaptic plasticity (Yamaguchi, 2000; ChiquetEhrismann and Tucker, 2004; Dityatev and Schachner, 2006). Tn-W is found abundantly in the developing bone and stroma of certain tumours (Chiquet-Ehrismann and Tucker, 2004; Tucker and Chiquet-Ehrismann, 2009). Tn-X is the first tenascin member shown to be clearly associated with the human connective tissue disorder Ehlers-Danlos syndrome (EDS; Burch et al., 1997). Patients with a Tn-X deficiency suffer from skin hyperextensibility, joint hypermobility and poor wound healing ability (Bristow et al., 2005). These symptoms are caused by the occurrence of abnormal irregular collagen fibres. Tn-X plays a role in collagen fibrillogenesis by directly binding to collagen (Mao et al. 2002; Minamitani et al. 2004). Mice with a Tn-X deficiency also showed skin symptoms comparable with those of EDS (Mao et al., 2002).

Interestingly, in an analysis of human single nucleotide polymorphisms, $\mathrm{Tn}-\mathrm{X}$ was reported to be significantly associated with schizophrenia (Wei and Hemmings, 2004; Tochigi et al., 2007). However, thus far, there have been no neuroanatomical reports on the involvement of $T n-X$ in schizophrenia. In the mammalian central nerv- 
ous system (CNS), Tn-X mRNA expression has only been shown in the rat meninges of the olfactory bulb (Deckner et al., 2000). Recently, we found novel Tn-X localizations in the adult mouse leptomeninges trabecula in the cerebral cortex and in the connective tissue in the lateral ventricle choroid plexus (CP; Imura and Sato, 2008). Our finding of $\mathrm{Tn}-\mathrm{X}$ localization in $\mathrm{CP}$, which produces cerebrospinal fluid (CSF), might be a key factor in the investigation of the association between CSF metabolism and enlarged ventricles in schizophrenia. Enlarged ventricles are typical structural abnormalities associated with schizophrenia (Staal et al., 1999). Furthermore, CP secretes biologically active molecules into the CSF for brain development, activity and protection (Strazielle and Ghersi-Egea, 2000; Brown et al., 2004; Thouvenot et al., 2006; Johanson et al., 2008). In these molecules, for instance, there is a brain-derived neurotrophic factor (BDNF), the gene expression level and polymorphism of which have been analysed in relation to the pathogenesis of schizophrenia (Buckley et al., 2007). One study reported that BDNF is able to stimulate $\mathrm{Tn}-\mathrm{X}$ expression in vitro (Takeda et al., 2005).

The validity and limitations of animal models (rodents and monkeys) for use in the study of schizophrenia have been discussed (Tordjman et al., 2007). The authors concluded that monkeys appear to be an interesting social interaction model, more so than rodents, because of their complex well-organized social structure. In addition to differences in social structure, the dopaminergic system of rats and monkeys is quite different (García-Cabezas et al., 2009), and dysfunction of the dopaminergic system is related to schizophrenia (Wang et al. 2008).

The CSF outflow system has been studied in some animal models (Kapoor et al., 2008). An anatomical difference in arachnoid granulations has been shown between rodents and monkeys (Krisch, 1988). Arachnoid granulations in monkeys are structurally similar to those in humans (Cooper, 1958; Krisch, 1988). In contrast, arachnoid granulations in rodents are similar to those of cats and dogs (Krisch, 1988). It is possible that $\mathrm{Tn}-\mathrm{X}$ localization in $\mathrm{CP}$ is different between rodents and monkeys.

Therefore, details concerning $\mathrm{Tn}-\mathrm{X}$ localization in monkey CP need to be clarified. In the present study, we compared the immunohistochemistry of
Tn-X in monkey $\mathrm{CP}$ with that in mouse $\mathrm{CP}$. Subsequently, to verify the reliability of $T n-X$ localization, we compared it with $\mathrm{Tn}-\mathrm{C}$ localization in corresponding regions using neighbouring sections.

\section{Materials and Methods}

Two male monkeys (Macaca mulatta) weighing 3.3 and $8.0 \mathrm{~kg}$ and five male ICR mice ( 11 weeks of age) were used as experimental animals in this study. The monkey tissues were a gift of $\mathrm{Dr}$ Kathleen Rockland (RIKEN Brain Science Institute). This study was conducted in accordance with of the official Japanese regulations for research on animals (according to institutionally approved protocols; RIKEN Institute and the Nippon Dental University, approval no. 07-04) and in accordance with the National Institutes of Health Guide for the Care and Use of Laboratory Animals (NIH Publication no. 80-23, revised 1996). The animals were perfused with $4 \%$ paraformaldehyde in $0.1 \mathrm{M}$ phosphate buffered saline after being deeply anaesthetized with nembutal as previously reported (Imura and Rockland, 2007; Imura and Sato, 2008). The monkey CP was carefully dissected from the lateral and fourth ventricles. Mouse brains were removed from the skull. The tissues were rapidly frozen by immersing them in a cold acetone solution with dry ice and were cut transversely or horizontally at $30 \mu \mathrm{m}$ with a cryostat. We used a previously described immunohistochemical to analyse Tn-X and Tn-C (Imura and Sato 2008). Briefly, the sections were processed for either Tn-X or Tn$C$ single immunoperoxidase staining (goat polyclonal anti-Tn-X antibody, catalogue no. sc-5498 or anti-Tn-C antibody, catalogue no. sc-9871; Santa Cruz Biotechnology, Santa Cruz, CA). The sections were incubated for 2 days at $4^{\circ} \mathrm{C}$. Finally, the sections were incubated with biotinylated antigoat secondary rabbit antibody (Vector Laboratories, Burlingame, CA) and visualized using avidin-biotin complex conjugated horseradish peroxidase ( $A B C$ Elite kits, Vector Laboratories) with diaminobenzidine. Routine controls for immunostaining were used during these experiments, by omission of the two primary antibodies. Photographs were taken as digital images with a digital camera (VB7010; Keyence, 


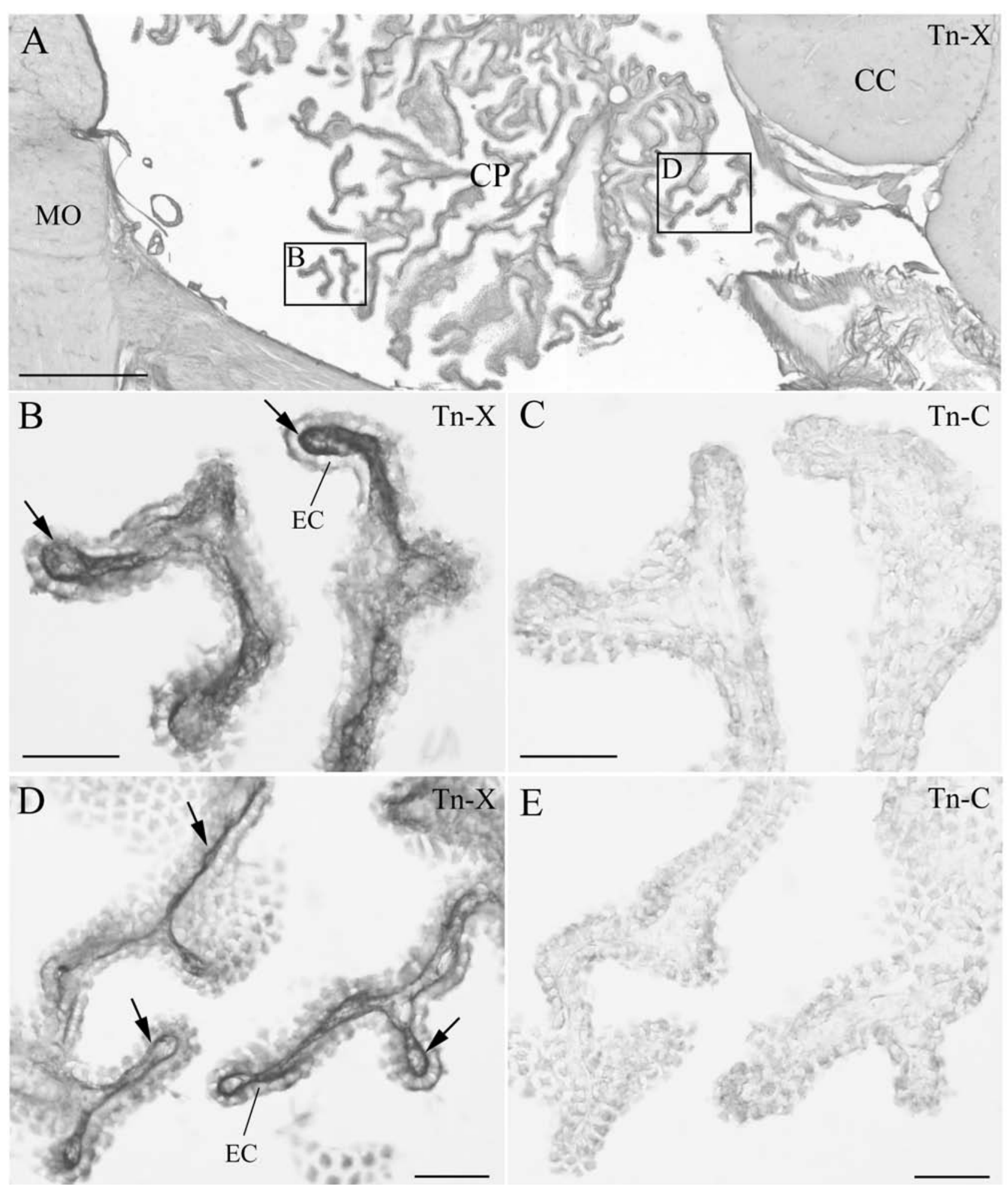

Figure 1 Immunohistochemistry (IHC) of tenascin-X (Tn-X) and tenascin-C (Tn-C) in monkey choroid plexus (CP) in fourth ventricle. (A) Low magnification of IHC of Tn-X in the lateral recess of fourth ventricle. Two boxed regions are re-photographed at higher magnification in B and D. (B, D) Tn-X positive fibrous structures are clearly observed within connective tissue of CP (arrows). (C, E) IHC of the Tn-C in neighboring sections to the $B$ and $D$. Tn-C positive fibrous structures are not detected within the connective tissue of CP. CC: cerebellar cortex, EC: epithelial cell, MO: medulla oblongata. Scale bars $=500 \mu \mathrm{m}$ in A, $50 \mu \mathrm{m}$ in B-E. 


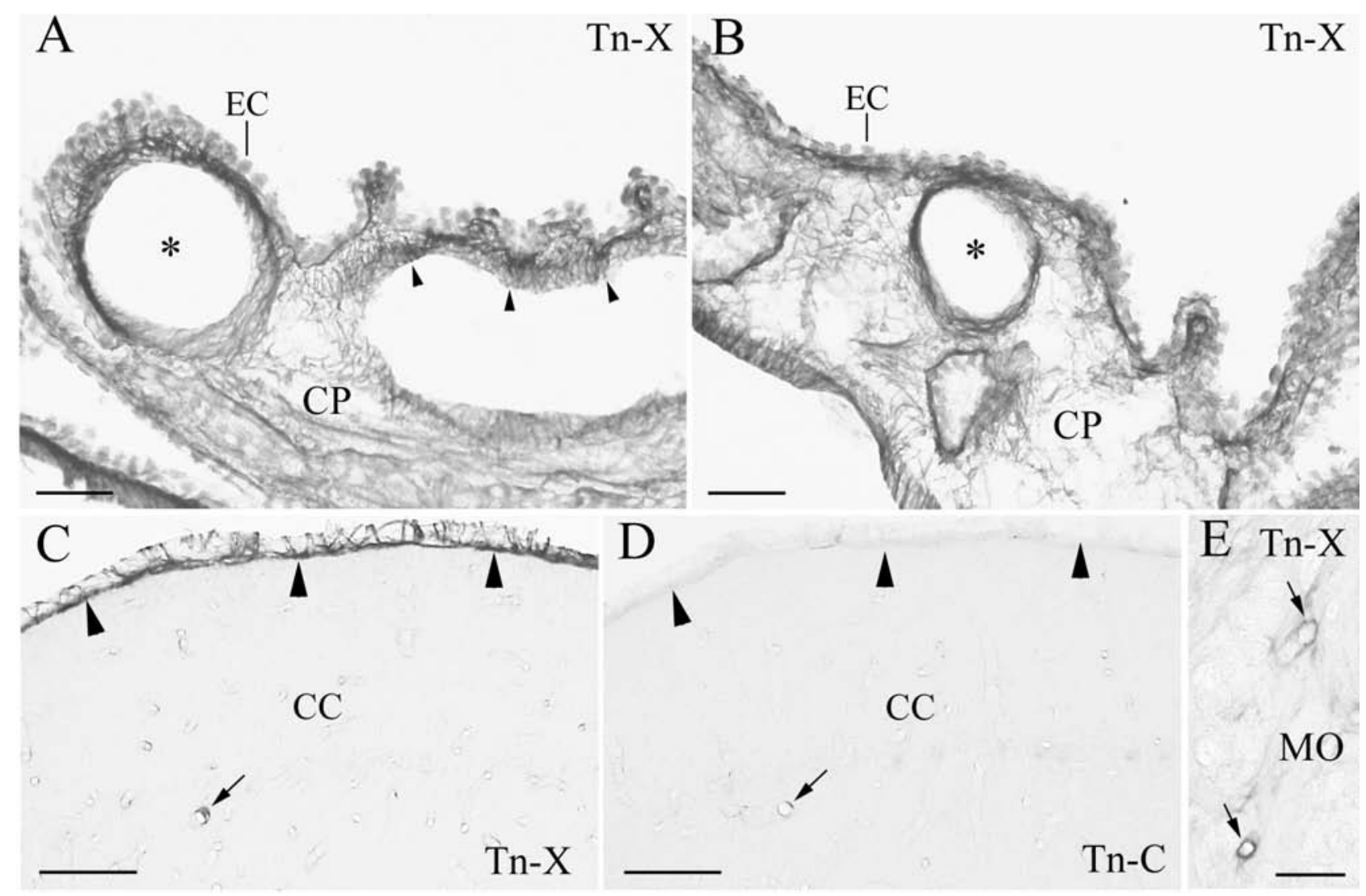

Figure 2. Tenascin-X (Tn-X) positive vascular and leptomenigeal structures in monkey choroid plexus (CP), cerebellar cortex (CC), and medulla oblongata (MO). (A, B) Tn-X positive fibrous structures are clearly observed around blood vessels (asterisks) and within the connective tissue of CP in lateral ventricle (arrowheads). (C) Leptomeninges on the CC is strongly Tn-X positive (arrowheads). An arrow indicates $\mathrm{Tn}-\mathrm{X}$ positive blood vessel. (D) Immunohistochemistry of tenascin -C in a neighboring section to the $\mathrm{C}$. Tn-C positive leptomeninges is not observed in the CC (arrowheads). An arrow indicates Tn-C positive blood vessel, which is corresponding to the Tn-X positive blood vessel in C (arrow). E: Tn-X positive blood vessels are also observed in MO (arrows). EC: epithelial cell. Scale bars=50 $\mu \mathrm{m}$.

Japan) equipped with a microscope (DM-RE; Leica Microsystems, Germany). The images were adjusted for contrast and/or brightness to match the real image using standard image software (Adobe Photoshop 5.5, Adobe, San Jose, CA).

\section{Results}

We observed Tn-X and Tn-C localizations in the fourth and lateral ventricles of monkey $C P$ (Figures 1 and 2A, B). Tn-X-positive $(T n-X+$ ) fibrous structures were clearly observed in $C P$ (Figures 1 and 2A, B) of both ventricles. The Tn$X+$ fibrous structures were restricted to the interstitial connective tissue in $C P$ (Figures 1 and $2 A$, $B$ ). In neighbouring sections, $\mathrm{Tn}-\mathrm{X}+$ fibrous structures were clearly confined to the connective tissue and did not colocalize with Tn-C in CP (Figure
IB, E). Tn-X was notably distributed around the blood vessels in $C P$. Dense $T n-X+$ fibrils surrounded blood vessels in $C P$ (Figure $2 A, B$ ). The presence of $\mathrm{Tn}-\mathrm{X}+$ and $\mathrm{Tn}-\mathrm{C}+$ was confirmed in some blood vessels in the parenchyma of the cerebellum and medulla oblongata (Figure 2C, E). These results were consistent with previous reports of vascular localization of $\mathrm{Tn}-\mathrm{X}$ and $\mathrm{Tn}-\mathrm{C}$ (Matsumoto et al., 1994; Hasegawa et al., 1997; Ballard et al., 2006). In addition, a leptomeningeal $\mathrm{Tn}-\mathrm{X}+$ trabecular structure was also observed in the cerebellum (Figure $2 \mathrm{C}$ ). In contrast, no leptomeningeal localization of $\mathrm{Tn}-\mathrm{C}$ was detected (Figure 2D). The $\mathrm{Tn}-\mathrm{X}+$ trabecula might correspond to the leptomeninges trabecula in the subarachnoid space (Haines, 1991; Imura and Sato, 2008). These observations in monkeys were similar to those of our previous findings of $T n-X$ localization in mouse CP of lateral ventricles and 
leptomeninges (Imura and Sato, 2008).

In the fourth ventricle of mouse $\mathrm{CP}, \mathrm{Tn}-\mathrm{X}+\mathrm{fib}-$ rils were restricted to the interstitial connective tissue and leptomeninges in the cerebellum, and no localization of Tn-C was observed (Figure 3 ). These findings in the fourth ventricle were homologous in the monkeys described above. Tn-X localization in CP was consistently confirmed using immunohistochemical techniques in the lateral and fourth ventricles of monkeys and mice.

\section{Discussion}

We observed Tn-X localization in monkey CP in the present study. These findings were not previously reported in primates.
In mouse $C P$, we confirmed our previous reports of $\mathrm{Tn}-\mathrm{X}$ localization in the lateral and fourth ventricles (Imura and Sato, 2008; present study). $\mathrm{Tn}-\mathrm{X}$ localization in both species was confined to the interstitial connective tissue in CP. Tn-X directly binds to type I collagen and regulates type VI collagen for collagen fibrillogenesis ( Mao et al., 2002; Minamitani et al., 2004). Collagen fibril in the CP connective tissue has been clearly observed in monkeys (Ling 1981). Furthermore, the connective tissue of CP consists of type I collagen in mice (Masuda et al. 1998) and of type VI collagen in humans (Roggendorf et al., 1988). Because of the presence of collagen fibres and the role of $T n-X$ in collagen fibrillogenesis, $T n-X$ localization in $\mathrm{CP}$ has a functional role, although

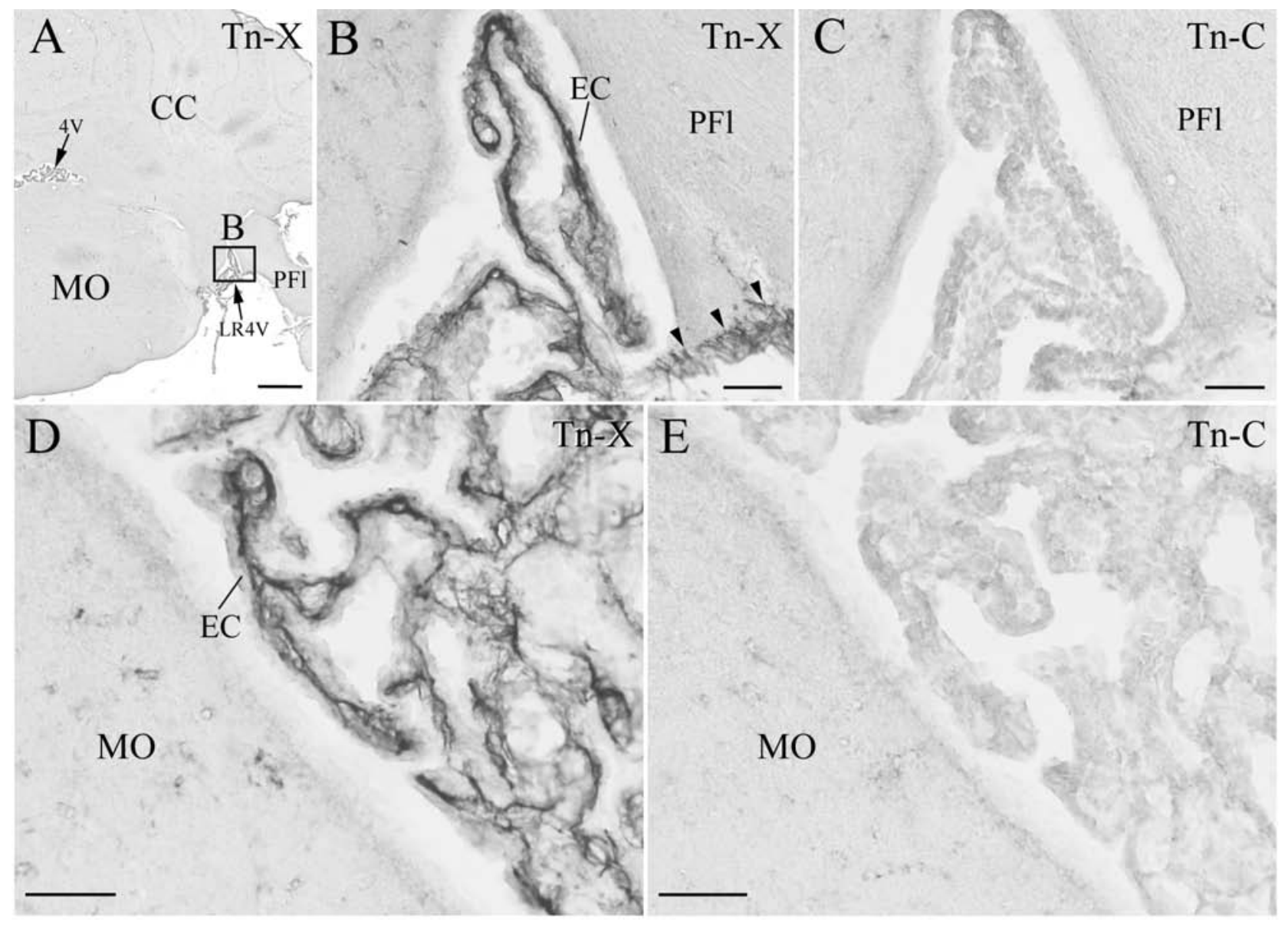

Figure 3. Immunohistochemistry (IHC) of the tenascin-X (Tn-X) and tenascin-C (Tn-C) in mouse choroid plexus (CP) in fourth ventricle. (A) Low magnification of IHC of Tn-X in a coronal section at fourth ventricle level. One boxed region is re-photographed at higher magnification in B. (B, D) Tn-X positive fibrous structures are clearly observed within connective tissue of CP. Three arrowheads in B indicate Tn-X positive leptomeninges on PFI (paraflocculus). The panel $D$ is photographed from an another section. (C, E) IHC of the Tn C in neighboring sections to the B and D. Tn C positive fibrous structures are not detected within the connective tissue of CP and leptomeninges. 4V: fourth ventricle, CC: cerebellar cortex, EC: epithelial cell, LR4V: lateral recess of 4V, MO: medulla oblongata. Scale bars $=500 \mu \mathrm{m}$ in $\mathbf{A}, 50 \mu \mathrm{m}$ in B-E. 
the type of collagen in monkey CP is unknown.

Tn-X deficiency is consistent with structural defects in human collagen. Patients with the connective tissue disorder EDS have been found to lack Tn-X (Burch et al., 1997); incomplete collagen fibere bundles are responsible for the symptoms. In addition to skin symptoms, vascular fragility and an abnormal perivascular structure have also been observed in EDS patients (Burch et al. 1997). In mouse and human blood vessels, Tn-X was confirmed by immunohistochemistry (Matsumoto et al. 1994; Hasegawa et al. 1997). Type VI collagen was observed in CP blood vessels in humans (Roggendorf et al., 1988). These previous reports might support our finding of dense $\mathrm{Tn}-\mathrm{X}$ localization around blood vessels in CP (Figure 2).

Recently, Tn-X was shown to be significantly associated with schizophrenia in a single nucleotide polymorphism analysis in humans (Wei and Hemmings, 2004; Tochigi et al., 2007). Previous reports and our present findings possibly indicate a novel important role of $\mathrm{Tn}-\mathrm{X}$ in the CNS. Tn-X interacts with vascular endothelial growth factor $B$ (VEGF-B) and BDNF in endothelial cells and enhances the function of VEGF-B in endothelial cell proliferation (Ikuta et al., 2000), and $T n-X$ expression is stimulated by BDNF (Takeda et al., 2005). In the nervous system, VEGF-B stimulates neurogenesis in the adult brain (Sun et al., 2006), and BDNF signalling is impaired in patients with schizophrenia (Buckley et al., 2007). The secretion of BDNF in $C P$ (Borlongan et al., 2004) and the strong expression of VEGF-B in CP of mouse embryo (Aase et al., 1999) suggest the possibility of an interaction between $\mathrm{Tn}-\mathrm{X}$ and these two molecules in CP. To clarify this hypothesized interaction of $\mathrm{Tn}-\mathrm{X}$ in $\mathrm{CP}$, further investigation is needed in future studies.

\section{Acknowledgements}

We thank Dr Kathleen Rockland of RIKEN BSI for donating the precious monkey tissue and members of the Department of Anatomy in NDU for their general cooperation.

\section{References}

Aase K, Lymboussaki A, Kaipainen A, Olofsson B, Alitalo K, Eriksson $U$. Localization of VEGF-B in the mouse embryo suggests a paracrine role of the growth factor in the developing vasculature. Dev Dyn 1999;215:1-25.

Adams JC, Watt FM. Regulation of development and differentiation by the extracellular matrix. Development 1993;117:1183-98.

Ballard VLT, Sharma A, Duignan I, Holm J M, Chin A, Choi R, Hajjar $\mathrm{KA}$, Wong SC, Edelberge JM. Vascular tenascin-C regulates cardiac endothelial phenotype and neovascularization. FASEB J 2006;20: 717-9.

Brown PD, Davies SL, Speake T, Millar ID. Molecular mechanisms of cerebrospinal fluid production. Neuroscience 2004; 129: 957-70.

Borlongan CV, Skinner SJM, Geaney M, Vasconcellos AV, Elliott RB, Emerich DF. Intracerebral transplantation of porcine choroid plexus provides structural and functional neuroprotection in a rodent model of stroke. Stroke 2004;35:2206-10.

Bristow J, Carey W, Egging D, Schalkwijk J. Tenascin-X, collagen, elastin, and the Ehlers-Danlos syndrome. Am J Med Genet C Semin 2005; 139: 24-30.

Buckley PF, Mahadik S, Pillai A, Terry Jr A. Neurotrophins and schizophrenia. Schizophr Res 2007;94:1-11.

Burch GH, Gong Y, Liu W, Dettman RW, Curry CJ, Smith L, Miller WL, Bristow J. Tenascin-X deficiency is associated with Ehlers-Danlos syndrome. Nature Genet 1997;17:104-8.

Chiquet-Ehrismann R, Tucker RP. Connective tissues: signalling by tenascins. Int J Biochem Cell Biol 2004; 36: 1085-89.

Cooper ERA. Arachnoid granulations in man. Acta Anat 1958;34: 187-200.

Deckner M, Lindholm T, Cullheim S, Risling M. Differential expression of tenascin- $C$, tenascin- $R$, tenascin- $/ \mathrm{J} 1$, and tenascin- $X$ in spinal cord scar tissue and in the olfactory system. Exp Neurol 2000;166:35062.

Dityatev A, Schachner M. The extracellular matrix and synapses. Cell tissue Res 2006:326:647-54.

García-Cabezas MA, Martínez-Sánchez P, Sánchez-González MA, Garzón M, Cavada C. Dopamine innervation in the thalamus: monkey versus rat. Cereb Cortex 2009;19:424-34.

Haines DE. On the question of a subdural space. Anat Rec 1991; 230: 3-21.

Hasegawa K, Yoshida T, Matsumoto K, Katsuta K, Waga S, Sakakura T. Differential expression of tenascin- $C$ and tenascin- $X$ in human astrocytomas. Acta Neuropathol 1997;93:431-7.

Hsia HC, Schwarzbauer JE. Meet the tenascins: multifunctional and mysterious. J Biol Chem 2005; 280: 26641-4.

Ikuta T, Ariga $\mathrm{H}$, Mtsumoto K. Extracellular matrix tenascin-X in combination with vascular endothelial growth factor B enhances endothelial cell proliferation. Genes Cell 2000;5:913-27.

Imura K, Rockland KS. Giant neurons in the macaque pulvinar: a distinct relay subpopulation. Front Neuroanat $2007 ; 1: 2$.

Imura K, Sato I. Novel localization of tenascin-X in adult mouse leptomeninges and choroid plexus. Ann Anat 2008;190:324-8.

Johanson CE, Duncan III JA, Klinge PM, Brinker T, Stopa EG, Silverberg GD. Multiplicity of cerebrospinal fluid functions: new challenges in health and disease. Cerebrospinal Fluid Res 2008:5: $1-32$

Kapoor KG, Katz SE, Grzybowski DM, Ludow M. Cerebrospinal fluid outflow: an evolving perspective. Brain Res Bull 2008;77:327-34.

Krisch B. Ultrastructure of the meninges at the site of penetration of veins through the dura mater, with particular reference to pacchionian granulations investigations in the rat and two species of newworld monkeys (Cebus apella, Callitrix jacchus). Cell Tissue Res 1988;251:621-31.

Ling EA. Ultrastructure and mode of formation of epiplexus cells in the choroid plexus in the lateral ventricles of the monkey (Macaca fascicularis). J Anat 1981; 133: 555-569.

Mao JR, Taylor G, Dean WB, Wagner DR, Afzal V, Lotz JC, Rubin EM, Bristow J. Tenascin- $X$ deficiency mimics Ehlers-Danlos syndrome in mice through alteration of collagen deposition. Nature Genet 2002; 
$30: 421-5$.

Masuda H, Hosokawa N, Nagata K. Expression and localization of collagen-binding stress protein Hsp47 in mouse embryo development: comparison with types I and II collagen. Cell Stress Chaperones 1998;3:256-64.

Matsumoto K, Saga Y, Ikemura T, Sakakura T, Chiquet-Ehrismann R. The distribution of tenascin- $X$ is distinct and often reciprocal to that of tenascin-C. J Cell Biol 1994;125:483-93.

Minamitani T, Ikuta T, Saito $Y$, Takebe G, Sato M, Sawa H, Nishimura T, Nakamura F, Takahashi K, Ariga H, Matsumoto K. Modulation of collagen fibrillogenesis by tenascin- $X$ and type VI collagen. Exp Cell Res 2004;298:305-15.

Roggendorf W, Opitz H, Schuppan D. Altered expression of collagen type VI in brain vessels of patients with chronic hypertension. Acta Neuropathol 1988;77:55-60.

Staal WG, Pol HEH, Kahn RS. Outcome of schizophrenia in relation to brain abnormalities. Schizophr Bull 1999;25:337-48.

Strazielle N, Ghersi-Egea JF. Choroid plexus in central nervous system: biology and physiopathology. J Neuropathol Exp Neurol 2000;59: 561-74.

Sun Y, Jin K, Childs JT, Xie L, Mao X0, Greenberg DA. Vascular endothelial growth factor-B (VEGFB) stimulates neurogenesis: evidence from knockout mice and growth factor administration. Dev Biol 2006;289:329-35.

Takeda K, Shiba H, Mizuno N, Hasegawa N, Mouri Y, Hirachi A,
Yoshino $H$, Kawaguchi $H$, Kurihara $H$. Brain-derived neurotrophic factor enhances periodontal tissue regeneration. Tissue Eng 2005; 11:1618-29.

Thouvenot E, Lafon-Cazal M, Dementtre E, Jouin P, Bockaert J, Marin P. The proteomic analysis of mouse choroid plexus secretome reveals a high protein secretion capacity of choroidal epithelial cells. Proteomics 2006;6:5941-52.

Tochigi M, Zhang X, Ohashi J, Hibino H, Otowa T, Rogers M, Kato T, Okazaki Y, Kato N, Tokunaga K, Sasaki T. Association study between the TNXB locus and schizophrenia in a Japanese population. Am J Med Genet 2007;144B: 305-9.

Tordjman S, Drapier D, Bonnot O, Graignic R, Fortes S, Cohen D, Millet B, Laurent C, Roubertoux PL. Animal models relevant to schizophrenia and autism: validity and limitations. Behav Genet 2007; 37:61-78.

Tucker RP, Chiquet-Ehrismann $\mathrm{R}$. The regulation of tenascin expression by tissue microenvironments. Biochim Biophys Acta 2009;1793, 888-92.

Wang M, Lee FJS, Liu F. Dopamine receptor interacting proteins (DRIPs) of dopamine dl-like receptors in the central nervous system. Mol Cell 2008;25:149-57.

Wei J, Hemmings GP. TNXB locus may be a candidate gene predisposing to schizophrenia. Am J Med Genet 2004;125B:43-4.

Yamaguchi Y. Lecticans: organizers of the brain extracellular matrix. Cell Mol Life Sci 2000;57:276-89. 
K. Imura and I. Sato 